\title{
La plage comme espace de l'écriture intime dans La vie pieds nus d'Alan Pauls
}

\section{The beach as a space for intimate writing in Alan Pauls' Barefoot life}

\author{
Mélanie MAILLOT ${ }^{1}$ [C]
}

'PhD Student, The University of Adelaide, Faculty of Arts, Department of French Studies, Adelaide, Australia

ORCID: N.C. 0000-0001-8706-8427

\section{Corresponding author:}

Mélanie MAILLOT,

The University of Adelaide, Faculty of Arts, Department of French Studies, North Terrace Campus, Napier builiding, Office 825, Adelaide 5005, Australia

E-mail: melanie.maillot@adelaide.edu.au

Submitted: 01.02 .2019

Revision Requested: 10.04.2019

Last Revision Received: 12.04 .2019

Accepted: 07.05.2019

Citation: Maillot, M. (2019). La plage comme espace de l'écriture intime dans La vie pieds nus d'Alan Pauls. Litera, 29(1), 19-40.

https://doi.org/10.26650/LITERA2019-0008

\begin{abstract}
RÉSUMÉ
Le motif de la plage a été exploré à maintes reprises en littérature : de Proust à Camus, elle a souvent incarné un lieu d'interactions entre l'expérience intériorisée d'un personnage et la réalité du dehors. Dans cet aller-retour entre l'intime et le collectif, Alan Pauls exploite la plage comme outil d'une écriture autobiographique, créant alors une réelle " phénoménologie » de la plage et révélant dans son texte une méta-littérature stratifiée et texturée, à l'image des grains de sable dans lesquels les pieds du baigneur s'enfoncent. La plage devient un espace multidimensionnel qui lui permet de décortiquer son identité plurielle et de se reconnecter aux souvenirs d'une enfance passée en Argentine dans les années soixante et soixantedix. Pauls met en place cette spatio-temporalité notamment à travers la mémoire visuelle et les jeux d'images, que ce soit directement par l'écriture ou par l'insertion de photographies et de références cinématographiques. La vie pieds nus s'offre à la fois comme une plongée dans les abysses de la pensée et une siesta sereine dans les sables mouvants de l'intime. Pauls nous emmène à la découverte de son moi dans un récit qui nous emporte comme la vague et nous laisser flotter dans l'écume de nos propres vies.
\end{abstract}

Mots-clés: Alan Pauls, écriture autobiographique, littérature argentine, philosophie, plage

\section{ABSTRACT}

The beach motif has been explored many times in literature: from Proust to Camus, it has often embodied a place of interaction between a character's internalised experience and the reality of the outside world. In this round trip between the intimate and the collective, Alan Pauls uses the beach as a tool for autobiographical writing, thus creating a real "phenomenology" of the beach and revealing in his text a stratified and textured meta-literature, like the grains of sand in which the swimmer's feet sink. The beach becomes a multidimensional space that allows him to dissect his plural identity and reconnect with the memories of a childhood spent in Argentina in the sixties and seventies. Pauls sets up this spatio-temporality particularly through visual memory and a play on images, whether directly through his writing or by inserting photographs and cinematographic references. Barefoot life is both a dive into the depths of the mind and a serene siesta in the quicksands of intimacy. Pauls takes us on a journey of discovery of his self in a story that takes us like the wave and lets us float in the spume of our own lives.

Keywords: Alan Pauls, autobiographical writing, Argentine literature, philosophy, beach 


\section{EXTENDED ABSTRACT}

The term 'beach read' has been associated with a number of pejorative meanings such as 'easy-reading' and 'fuss-free books'. This study aims to deconstruct these stereotypes in order to show how a book, undercovered by the theme of the beach, can carry a metaphysical reflection that touches both the writing process and the construction of an identity. Alan Pauls retraces his teenage journey by taking the road back to the Argentine beaches he frequented at the time, with the aim of rediscovering himself as a writer but also and above all as a man. The main purpose of this article is therefore to question this beach space and see how it becomes the place par excellence of Pauls' intimate writing. The interpretative guidelines given in this study provide a better understanding of how a real "phenomenology" of the beach is born in this autobiographical essay.

The first part of the article focuses on the development and exploration of identity through the beach motif. Three identity perspectives are considered. First of all, it is Pauls' Latin American historical and cultural heritage that is being explored, including the "beach culture". After that, it is his literary identity that is analysed. Pauls draws inspiration from other beach writers and builds in his text a well-crafted intertextuality that we decode. Moreover, this part reveals his writing choices and explains his attraction to literary hybridity and the mixing of genres. The last identity perspective concerns the style of Barefoot life, which, like the beach, is composite, granular and multiform. The stylistic analysis that is carried out highlights the concept of writer" of the intimate" and the process of identifying Pauls as an autobiographer.

The second part focuses on the meta-literature that emerges from the text and shows how it is closely linked to the conception of intimate writing, but also to metaphysical reflection. Life ethics are gradually being formed through salty and sandy reminiscences. The beach is given as a metaphor for an autobiographical literature where the writer can meditate on his existence. This book therefore also questions this "exposure" and the dialogue between the intimate and the collective represented by the beach, which is necessary in the process of transmitting universal thought. In the meanders of this reflection, symbolised by the place of the beach, the self is fragmented and manipulated to such an extent that it becomes a Freudian subject. The text therefore also goes through the concept of strangeness and illustrates, through the literary processes at work, a movement of distancing from the 
"I". To make sense of this strangeness, the narrator relates to his sensory orientation, constructing in the work a synesthesia that will make it possible to define a writing of memory.

The last part focuses on the cult of the image and the importance of the visual. This approach allows to link identity to its plural nature and to complete a text that is intended to be polymorphic. We show that Pauls uses different modalities to visually translate the beach as a pivotal element between the external environment and the narrator's intimacy. Graphic precision is one of these modalities: from typographical choices to mises en abyme, as well as through ekphrasis, Pauls makes the beach a place where the self can be observed and where the writer can build this writing of the intimate as one would build a sand castle. We also explore his particular use of photography and cinematographic references, creating a "cinematographic" writing that gives access to a multiplicity of experiences, including sexuality and violence. This approach also helps to understand how cinema has strongly influenced Pauls as a writer. Finally, we show how the beach is represented as a "white screen" and how it becomes a symbol of artistic freedom and liberation of the subject. As he contemplates his own existence, the narrator invites the reader under the umbrella of this symbolic beach and the strength of Pauls' text then takes effect: the writing of the intimate takes us like a wave towards the sea of our own memories.

In conclusion, Alan Pauls' Barefoot life is a hybrid book, between essay and autobiography that unites under the power of one and the same motif: the beach. Our study was able to show the importance of the workings between the intimate and the collective, between writing and visual arts and finally between philosophy and literature. This book defines Pauls' identity as a man, a writer and a philosopher, which appears ultimately plural and changing, like the tides. 


\section{Introduction}

La vie pieds nus est un livre "de plage », non pas au sens de légèreté, mais au sens de profondeur et d'épaisseur. Le thème de la plage y est présenté comme une plaque tournante, qui relie et mouvemente le récit autobiographique et la réflexion philosophique. Alan Pauls déroule sa serviette sur le sable chaud pour nous parler de son enfance, sans cesse avalée puis rejetée par le mouvement des vagues, récit d'une beauté mélancolique mais aussi récit de découverte à travers le concept du cinéma drive-in, où la pensée philosophique se forme grâce à la connexion entre la plage et le monde des images. L'écrivain argentin y maintient un équilibre parfait entre narration et digression pour dire quel adolescent il était et quel écrivain il est, entre pensée et perception. «Pour moi, l'intime c'est le monde » a-t-il dit dans un entretien (Millet, 2008, p. 63). De ce fait, à l'image de la mer, il présente un univers où les traits s'effacent, où l'infinité prend place sur la petitesse de sa personne, où le tout enveloppe l'unique, sentiment qu'il essaye de retranscrire en enfermant ses souvenirs dans un livre. Dans la profondeur de son texte, invisible à la surface mais bien présente, il donne une certaine image de l'écrivain dans sa complexité humaine. II n'est plus uniquement auteur, il est plusieurs personnes à la fois. Ainsi, il est de circonstance de se demander comment Alan Pauls déroule, à travers son essai autobiographique, une psychologie de l'écriture à travers le thème de la plage. Premièrement, il s'agira de révéler l'écriture de Pauls comme expression d'une identité. Deuxièmement, nous verrons en quoi cette même écriture s'ouvre sur une métalittérature. Enfin, ce déploiement étant mis en lumière à travers le culte de l'image, il sera de circonstance d'y consacrer le dernier axe de réflexion.

\section{La perspective identitaire}

L'acte de l'écriture intime n'est pas tout à fait similaire à l'autobiographie. Le premier est un dynamisme fondé sur une multitude de paramètres textuels personnels tandis que le second est un genre littéraire. De la même manière, écrire l'intime ne veut pas forcément dire écrire sur soi, mais plutôt écrire sur le particulier, le spécifique voire le typique dans un espace délimité par la fantaisie de l'auteur, tandis que l'autobiographie implique plus de formalité, selon les exigences et les codes du genre, comme définis par Philippe Lejeune dans Le Pacte autobiographique. C'est pourquoi Alan Pauls choisit l'hybridité. Elle lui permet d'exprimer une identité à la fois familière et altière, démarche qui apparaît rapidement comme complémentaire 
et essentielle au déchiffrage de son individualité. Cette identité se compose de trois aspects dominants. Le premier n'est autre que I'héritage latino-américain qui soustend le texte et apparaît comme la toile de fond de Pauls. Au sens de pays, au sens de culture mais aussi au sens du social, sa conception de l'intime est amplement politique. Son narrateur-personnage construit sa relation au monde par le spectre de l'intime, c'est-à-dire que le monde intérieur et le monde extérieur se rejoignent car ils sont tous deux agonistiques. En effet, il s'agit d'une histoire d'adolescence dans les années soixante-dix en Argentine. Indéniablement, Alan Pauls met côte à côté Histoire et mémoire, dans une démarche qui relève de l'interrogation et de la réflexion sur le passé, plutôt que d'une tentative de recomposition de ce dernier, comme l'explique Gersende Camenen (2012). L'histoire qu'il écrit s'entoure donc des discours socio-politiques de son époque, renvoyés en miroir au lecteur comme clé de lecture des éléments qui fondent l'intimité de l'auteur. Le cadre spatio-temporel en est le premier indice: d'une part est présentée l'influence politique sur les arts et la culture, comme le montrent la mention du « Plan Galopant » de la ville de Gesell, qui est un plan d'aide à l'achat immobilier travaillant stratégiquement avec le showbusiness de Buenos Aires (p. 28) et l'impact sur la culture de la plage, qui est décrite à différents passages du texte, où elle rime avec " magazines people », " presse fitness », " magazines porno soft » et «Brigitte Bardot argentine » (p. 75) et accueille principalement des " séducteurs », des « starlettes », des " parlementaires » et des " mannequins », dans leur monde de superficialité extrême (grosses voitures, sponsors de marques, discothèques, etc.) (p. 85). Cette culture stérile est résumée à travers les expressions $d^{\prime}$ " expérience érotico-politique » et de " petite société sans Etat ni marché » (p. 81). D’autre part est présenté l'esprit révolutionnaire, jouant le rôle de décor, comme par exemple l'affiche publicitaire politique assortie d'un «Chiruchi gros pédé » (p. 11), Miguel Mateos décrit comme un « outsider du rock argentin » (p. 10), la mention du cri de guerre du mai 68 français (p. 35) et les groupes de hippies anticapitalistes dans leurs vans Volkswagen partant en voyage pour « faire valoir leurs droits » (p. 21). C'est donc dans ces conditions historico-politiques que le narrateur grandit et forge son identité. C'est à ce moment qu'intervient la découverte du cinéma drive-in, « divertissement nord-américain » rendu possible grâce à «l'union de l'industrie automobile et de celle du cinéma » (p. 17). Ainsi, il faut comprendre que le contexte historique a une influence indéniable sur l'adolescent puisque cette expérience du cinéma jouera un rôle crucial dans sa conception du monde. D'autres exemples comme celui-ci peuvent être relevés, à savoir l'effet de patchwork qui semble faire pression au sein de la culture argentine, qui se mêle alors au « strudel », à 
la « Fiat 600 », à l'architecture des « chalets », aux « sabots bavarois » et à la promotion des «mythologies nordiques» (pp. 24-25), mais qui rejette d'autres formes de culture par une censure sévère, notamment du cinéma et de la presse " qui n'arrivaient jamais jusqu'aux kiosques de Buenos Aires » (p. 26). Pour clore cette réflexion sur I'héritage latino-américain, il faut figurer la plage et le cinéma comme des moyens pour l'adolescent de relier son intimité à l'univers public. En ce sens Camenen fait remarquer que « l'écrivain redonne tout son sens, subjectif et politique, à l'intime, comme série d'expériences qui façonnent le sujet (personnage et lecteur), dans son rapport aux autres et interdisent une adhésion pure et simple au système de valeurs promu par la société en place » (2012). Cette réflexion s'illustre parfaitement dans le texte, toujours à travers le thème de la plage. Elle est d'abord le lieu de la « liberté, tolérance, sociabilité égalitaire » (p. 47), puis elle devient lieu de "résistances, stoïcismes, capacités de travail et mystiques » (pp. 93-94). L'ambivalence est donc une notion inhérente à la plage, ceci étant créé par l'impact culturel et social, qui dicte une pensée de la plage. Mais comme le dit Camenen, Alan Pauls se dresse contre l'adhésion au diktat de la plage, en la transformant, par la force de l'intime, en en lieu " [immunisé] contre toute intervention extérieure, [livré] à l'inertie d'une logique propre mais insaisissable » (p. 48). En d'autres termes, l'intime devient l'élément médiateur face à l'affaire publique et la futilité extérieure.

Le second aspect de cette écriture intime réside dans l'identité exclusivement littéraire. En tant qu'écrivain, il est évident qu'il ait trouvé des modèles dès son plus jeune âge. En effet, au fil de la lecture, le texte se révèle comme une source riche d'intertextualité. Tout commence dans le développement du plaisir de la lecture : « je me souviens d'avoir acheté [...] les premiers livres que je choisis moi-même, La fin d'un jeu, Tous les feux le feu et Les Gagnants, qui sellèrent pour toujours une capricieuse alliance entre Cortázar et la plage » (p. 27). Il explicite donc une connexion entre l'expérience physique de la plage (maillot de bain, sable, sel) et l'expérience mentale des mots sur la page. À partir de là, l'intertextualité prend une autre direction interprétative : la littérature est généralisée au concept de la plage. Il fait référence à différents auteurs, mais toujours en relation avec ce même concept : Diderot et sa réflexion sur la côte hollandaise (p. 48), Proust et la plage de Balbec (p. 71), la littérature et la culture antiques à travers la mythologie et sa légende de l'eau, de Vénus aux baiae sous l'Empire romain (p. 45), mais surtout la mention de L'Étranger de Camus (pp. 34-35), qui est très certainement la référence la plus importante. En effet, l'adolescent apparaît presque comme une autre version du personnage de 
Mersault, lui-même isolé de la société à cause de son rapport sensuel au monde, d'une conception en diffraction de la plage, qui détient un pouvoir d'une étrangeté inexplicable. Il y a donc identification au personnage littéraire, de manière consciente et/ou inconsciente. Cette intertextualité est encore poussée à ses limites à travers certaines références cachées du texte où Pauls semble se citer lui-même. C'est le cas par exemple lorsqu'il fait référence à la couleur blonde de ses cheveux, juxtaposée aux termes «nazie » et " enfant allemand ». Cela rappelle sans aucun doute son autre texte, Histoire des cheveux, où le protagoniste est plongé dans une obsession capillaire et un fétichisme certain, le renvoyant sans cesse vers ses souvenirs d'enfance. Mais le concept d'identité littéraire ne s'arrête pas là, puisqu'il se rattache également à un mélange des genres, comme par exemple lorsqu'il inclut de la poésie : "C'était un soir / au coucher du soleil / le soir où tu fus mienne / le soir où je te vis, mon amour » (p. 32), ou qu'il vire au genre historique: « le bikini, le maillot de bain que Jacques Heim et Louis Réard inventèrent en 1946 [...] et qu'ils baptisèrent du nom d'une plage située dans le Pacifique Sud », puis au genre satirique dans la foulée: «l'atoll de Bikini - où, tout juste trois semaines auparavant, avait explosé la première bombe atomique de l'après-guerre ", et plus encore, au genre de l'essai tout de suite après, avec une parodie de référence savante: « Diana Vreeland n'aurait pu mieux définir l'impact que cette invention allait avoir sur la discrétion du corps: «La seule chose que ce vêtement ne dit pas d'une femme, c'est le nom de jeune fille de sa mère» affirma-t-elle » (p. 44), le tout étant toujours en relation avec l'univers de la plage. Alan Pauls peut donc être poète, historien ou satiriste. Similairement, il donne sa conception du personnage comme création littéraire selon cette même vision. Il parle de « virus dramatique », de "personnage principal » dans la «fiction de la plage », où il n'est possible de se distinguer qu'en étant soit un « héros», soit une « victime», soit un « idiot» (p. 38): la plage devient un théâtre. Enfin, l'identité littéraire jaillit aussi à travers I'héritage littéraire latino-américain, comme le prouve les similitudes avec l'écrivain Roberto Bolaño, comme l'effet de récit-puzzle où les parties sont détachables et interchangeables, le mélange des genres (par exemple le mélange de récit narratif, journal et monologues dramatiques dans 2666), la présentation d'un territoire de fiction, ici la plage (chez Bolaño, il s'agira des déserts du Sonora par exemple), la teneur satirique en relation avec la condition contemporaine. De la même façon, il se rapproche également de Jorge Luis Borges, qui a expliqué dans une interview que son but était de créer une fusion du mode fictionnel et du mode de l'essai (BellVillada, 1999, p. 58), faisant de lui le fondateur d'un genre nouveau en Amérique latine. Tout d'abord, il s'en rapproche à travers ses autres textes et tout 
particulièrement avec El factor Borges, mais aussi dans ce texte-ci à travers l'usage de la polygraphie et de l'hybridité. Enfin, la volonté de fractionner le texte littéraire, commune aux écrivains latino-américains du XXe siècle (Aubague, Franco, \& LaraAlengrin, 2009, p. 13), transparaît à travers un choix esthétique, celui de la fragmentation en action dans le récit, qu'il est à présent temps d'exposer.

Le dernier aspect de l'écriture intime n'est donc rien d'autre que le style individuel de l'auteur, en relation à ce qui vient d'être présenté. Alan Pauls se dévoile comme adolescent, comme adulte et comme écrivain, à travers la subtilité de sa plume. Son écriture dessine sa personnalité. Comme annoncé précédemment, I'un des traits distinctifs du texte réside dans l'apparence de fragmentation, de séparation. En effet, il utilise de nombreux procédés littéraires participant à cette esthétique, notamment l'oxymore et les oppositions d'idées, par exemple : la « petite vague miséricordieuse » contraste avec la « terre ferme mais humiliante » (p. 39), ainsi que les énumérations, qui viennent hacher la continuité du texte par une abondance de virgules, et cela à l'image des idées qu'il exprime. Le passage clé qui illustre cette idée est la description qu'il fait du sable : «Il est fait de déchets : débris de rochers, de récifs, de coraux, d'os, de coques, de plancton » (p. 31). Tout comme son style, le sable est «impur » et composite. Néanmoins, il n'en est pas moins poétique, comme le montre son agilité avec les allitérations : " un petit miracle d'euphonie monosyllabique et peut-être même l'annonce d'un érotisme sournois qui serpente dans cette succession de sifflantes » (p. 54). Ici, non seulement il créé un effet sonore de glissement avec la répétition du son [s], mais il créé en plus une « mise en abyme » métalittéraire de cette allitération, puisque le sujet de la phrase elle-même est la phonétique (d'où les termes « monosyllabique » et « sifflantes »). Il se complète également à travers l'idée d'un style savant, comme lorsqu'il utilise le latin: « otium cum dignitate » (p. 45), « in absentia » (p. 60 ), qui vient radicalement s'opposer à un sens de l'humour tout à fait unique et très développé au sein de l'œuvre, selon différents procédés tels que le détournement linguistique: « spécialistes ès plage » (p. 46), le registre familier: « partouze sauvage » (p. 49), « forniquer » (p. 64), I'usage du cliché: « C'est là une des fatalités qui condamnent la plage au kitsch: peu de scènes sont aussi ridiculement métaphoriques que la sortie de l'eau » (p. 58), la référence littéraire: «Sisyphe de plage » (p. 97), la description familière: « [le sable] faisant des leurs dans les entrejambes de Burt Lancaster et de Deborah Kerr » (p. 55), « ceux qui reviennent de la côte avec leurs valises pleines de prouesses génitales » (p. 74), et les comparaisons inédites: « aussi dépourvues de couleur locale et de glamour qu'un village de vacances pour enfants déshérités » ( $p$. 
60). Par ailleurs, ce n'est pas seulement l'écriture qui le définit comme personne, mais également la littérature en soi. En effet, il semblerait qu'il partage une relation toute particulière avec Hemingway, qui a lui aussi écrit un récit autobiographique auquel Pauls fait référence : Paris est une fête. De premier abord, cela semble très impersonnel, puisqu'il s'agit de l'œuvre de quelqu'un d'autre. Mais le rôle qu'elle joue dans la vie d'écrivain de Pauls est très précieux : elle lui permet de trouver sa voie/voix en tant qu'auteur de l'intime, comme il le précise en ces termes:

la politique d'Ernest Hemingway consistant à écrire dans les lieux publics, bars, terrasses de cafés, restaurants, halls d'hôtels [...] m'avait enchanté dès que je l'avais découverte, en lisant Paris est une fête, et que je tentai de m'approprier aussitôt, sans faire l'effort de me demander si la frénésie intime et maladroite que je confondais alors avec la pratique de la littérature était compatible avec l'exhibitionnisme et la nonchalance publiquement préconisés par Hemingway. (pp. 90-91)

Ainsi, il révèle ses choix d'écrivain et affirme quelques lignes plus loin : « mortellement blessé dans ma double sensibilité d'amoureux de la plage et d'écrivain : "C'est comme ça que doit être ma vie, comme ça et pas autrement» (p. 91). Cette subtilité ajoute quelque chose d'unique : il ne s'identifie plus simplement comme écrivain, mais comme écrivain de l'intime. Finalement, il ne donne pas accès à tous les aspects de sa personnalité. Ce qu'il veut transmettre avant tout, c'est son identité en tant qu'autobiographe, plus que l'autobiographie en elle-même. Il maintient le reste du temps, une identité qu'il tente de définir certes mais qui reste floue : « la plage est un contexte si fort, qui contraste tellement avec la vie qu'on peut y percevoir les identités de façon altérée [...] les deux espaces entretiennent d'une certaine façon une relation de fiction réciproque » (p. 63). Ceci constitut l'aveu de ne pas dire l'authenticité de l'identité. Puisque son récit est à propos de la plage, son identité ne peut être révélée que sur le mode de la diffraction. Lui-même, à travers son écriture, tente de comprendre quelle est sa place dans le théâtre du monde : « Mais quelle est ma scène? » (p. 112).

\section{Naissance d'une métalittérature}

L'écrivain, celui qui raconte sans cesse des vies de fiction, se retrouve à questionner la fiction de sa propre vie. En effet, l'écriture intime, si différente parce qu'elle prend pour sujet et objet l'individualité de son auteur, l'amène à développer une réflexion 
sur l'acte d'écriture lui-même. La littérature devient métalittérature, le moi de Pauls étant à mi-chemin entre les deux, précisément au centre de la réflexion, d'une part par sa qualité d'homme et d'autre part par sa qualité d'écrivain. C'est pourquoi il développe dans un premier temps des aspects métaphysiques, entre l'homme et la littérature, la plage étant le lieu de rencontre entre les deux. Le moi de Pauls trouve une identité à travers la plage, qui apparaît à la fois comme un tout et comme un assemblement de milliers d'éléments, sans lesquels le tout n'existe pas. Une réelle métaphysique de la plage est créée tout au long du texte et se décline selon plusieurs niveaux. Tout d'abord, la plage, tout comme l'écrivain, a une voix : « que la plage [...] soit minimaliste ne signifie pas qu'elle soit muette ni même laconique : la plage murmure et parle » (p. 34) et elle se suffit à elle-même: « mais en elle contenant et contenu, support et surface, paraissent indiscernables, comme s'ils étaient faits d'une même matière » (p. 34), au contraire de l'écrivain cette fois-ci, qui doit être en relation avec d'autres entités (papier, plume, éditeur, lectorat). On comprend donc que la plage est perçue comme une finalité inaccessible pour l'homme. Ensuite, la plage est le lieu de l'authenticité où « il n'y a pas de double-fond où se cacher, aucune zone d'ombre » (pp. 34-35). Ceci ne peut être interprété comme un gage de vérité, mais plutôt comme un gage de valeur : la littérature n'est pas faite pour dire la vérité, elle est faite pour dire des évidences, soit dont on ne s'était pas rendu compte, soit dont on avait trop bien conscience pour ne pas les dire. Enfin, la plage est un paradoxe : elle est « à la fois ce qui était là avant et ce qui viendra après, le début et la fin, ce qui est encore intact et ce qui est déjà pulvérisé, la promesse et la nostalgie » (p. 33), elle a à la fois une logique binaire simple et une logique « équivoque, tortueuse et même spectrale » (p. 88). Pauls rappelle également les mots de Camus, qui décrit la plage comme « une bouche ou une blessure » (p. 34), c'est-à-dire comme celle qui embrasse, ou celle qui blesse. De ce fait, une analogie se construit entre la plage et la figure de l'écrivain. Ce phénomène ouvre une réflexion métaphysique sur la condition humaine, à trois niveaux distincts qu'il faut développer. Premièrement, il s'agit du niveau théologico-culturel : la culture judéo-chrétienne transforme la plage hédoniste du monde romain en scène de désolation et par extension, de lieu du péché : «Irrégulière et floue, la ligne du rivage (c'est la limite que l'homme ne doit pas franchir) et l'océan, diabolisé par la mythe du Déluge, se change en instrument de chaos et de destruction » (p. 46). Ainsi, la plage permet à l'homme de réfléchir sur sa destinée voire même ses croyances. Pour Pauls, écrire sur la plage et faire l'expérience de la plage, c'est réfléchir sur le sort ultime de l'homme après sa mort et plus encore, sur celui de l'univers après sa disparition : 
la plage, espace eschatologique par excellence, recèle sous son air de tabula rasa les valeurs d'une ère primitive qui précède l'histoire et tous les traits d'un décor posthume qu'une catastrophe naturelle ou le coup de patte d'une force destructrice aurait réduit à ce qu'il y a de plus élémentaire: un paysage de ruines et de décombres microscopiques. (p. 33)

L'écriture de la plage révèle l'intime dans ses considérations eschatologiques individuelle et universelle. Deuxièmement, il s'agit du niveau de violence, où la plage devient une arène : "le lieu critique où les factions ennemies se regardent souvent pour la première fois droit dans les yeux, et c'est de ce fait un lieu de violence et un champ de bataille » (pp. 44-45). La plage devient métaphore de la littérature. À partir de ce point de vue, l'écriture devient le lieu de confrontation de l'écrivain avec luimême. Ecrire l'intime, c'est se faire violence, se confronter peut-être aux parts obscures de l'individualité ou de l'histoire personnelle, transformant ainsi la faiblesse en force. Il s'agit donc d'un exercice consistant à « déposer des mots sur le papier et à les prendre pour des compagnons, voire des confidents qui pourraient éclairer un peu plus la part d'ombre qui noircit et menace les lisières de [l'existence] » (Glykos, 2009, p. 26). L'écriture, par la confrontation, où la plage blanche devient un miroir, puis par l'acceptation, devient un remède aux maux. La littérature révèle un pouvoir thérapeutique. Troisièmement, il s'agit du niveau sexuel, plus largement exploité que les autres. Ce n'est pas Pauls qui met en place cette mythologie érotique de la plage, elle est bien présente à travers la culture et les sociétés, qui vivent au rythme du Sea, sex and sun. Cependant, la plage produit chez Pauls un effet bien plus profond sur son intimité et son identité sexuelle. En effet, la plage n'a rien d'érotique pour lui, si ce n'est à travers la culture. Son argument principal est qu'un décor de forêt, où règne fertilité, bourdonnement et humidité, serait plus propice à éveiller une tension sexuelle (p. 54). Pour lui, le caractère sexuel réside dans la perception. La plage est sexuelle parce qu'elle est rattachée au concept de naissance, thème infiniment pertinent pour un récit autobiographique. Cela désigne à la fois la naissance de l'homme (Vénus sortie des eaux) et la naissance du désir sexuel : « [La plage] est érogène parce qu'elle choisit de montrer non pas la consommation de l'acte sexuel, mais la naissance d'un objet du désir unique et mythique » (p. 59). Il se place dans la métaphysique sexuelle plus que dans la réalité physique du sexe, comme il le précise plus loin dans le texte: « la libido qui l'anime est moins dirigée vers des objets précis que vers des formes de vie utopiques » (p. 80), au contraire du reste de la société, qui semble concevoir la sexualité dans le règne du palpable. Il conclut que la nature en 
société, au lieu d'être considérée pour ses textures, couleurs et formes, n'est qu'un prétexte pour développer un culte de la vitalité, au sens de virilité pour l'homme et de fécondité pour la femme. Ainsi la plage, comme lieu de rencontre entre l'intime et le public, devient révélatrice d'une vision épistémique de la subjectivité, où l'individu et notamment l'enfant, peut réfléchir sur ce qui fait le vrai et le faux et de découvrir la différence entre la pensée intime et la pensée étrangère, c'est-à-dire la pensée de l'autre. Cette théorie de la pensée, selon André Carel, a une genèse qui entre en résonance avec l'image du corps (2004, pp. 3-4), et plus précisément dans le registre anal, selon les précisions de Graber (2004). Plus encore, Carel mentionne deux stades distincts du psychisme, qui peuvent expliquer la conception-perception d'Alan Pauls adolescent, par rapport à la plage : le premier stade est une ouverture théorique au monde de l'autre [sur la plage], mais toujours en considérant l'autre comme objet, ou comme un "moi non-moi », d'où l'attitude de rejet sexuel de l'adolescent. Le second stade va au-delà du narcissisme et devient copernicien, l'autre devient sujet (et non plus objet) créant ainsi un groupe où les trajets pulsionnels personnels s'enchevêtrent. C'est ce qu'il appelle précisément l'intersubjectalité (Carel, 2004, pp. 6-7). Par conséquent, en termes de métalittérature, la conception sexuelle de la plage offre une compréhension du psychisme de l'écrivain : l'espace intime est le jardin secret de l'auteur, que Pauls mentionne brièvement : « une expérience néfaste que je garde enfermée en moi à double tour » (p. 53) ; l'espace public, qualifié par la transparence, est le livre comme objet physique dans le monde ; l'espace privé est la relation entre l'écrivain et le lecteur, qu'il est dès à présent nécessaire d'aborder plus en détails.

La transmission fait effectivement partie de la réflexion métalittéraire et elle permet de passer de l'intime au collectif. D'un point de vue très basique, l'écriture constitut la matérialité d'un premier passage, celui de l'intime vers son contraire, à travers la publication, et d'un second passage, celui de l'histoire intime à une histoire universelle, au sein des littératures du monde. Plus précisément, l'écriture intime comme moyen de transmission illustre le passage du dedans vers le dehors de soi. Alan Pauls transmet cette idée implicitement dans son œuvre, en faisant usage de paramètres divers tels que le lien à la parenté: « toute main adulte que nous surprenons pendant près de notre tête peut être celle de notre père (poils sur l'avant-bras, montre, cigarette) ou de notre mère (ongles, vernis, lunettes de soleil), et une main peut appartenir à n'importe qui » (p. 36), les champs lexicaux: « confuses », « s'éloigner », « inconnu » (p. 37), l'idée de mouvement de masse: "Les éléments des groupes s'attirent, s'approchent, se joignent, se mêlent, se séparent. Vivre sur la plage n'exige qu'une condition, et cette condition 
est mystérieusement quantitative: il faut s'agréger » (p. 74), la confusion des identités: " j'ai moins le souvenir des visages et des noms, les deux signes privilégiés d'identification individuelle, que d'une sorte de mouvement continu de flux et de reflux » (p. 77) et enfin, la mise en évidence de l'individualité, comme par exemple à travers sa théorie du freak qui ne se mélange pas aux autres (p. 86) et qu'il exploite plus en détails vers la fin du livre en parlant de « communion non adhésive, dans laquelle les choses et les êtres peuvent se rencontrer et entrer en relation sans être obligés de se confondre les uns avec les autres» (p. 111). Cette idéologie fait naître une nuance nouvelle au sein de la réflexion métalittéraire : l'univers public accorde de la reconnaissance au monde intime de l'auteur, idée que font ressortir Goetgheluck et Conrath dans leur article sur l'intimité face à la collectivité :

Si, aujourd'hui, les drames internes se jouent sur la place publique, ce n'est pas seulement un effet de la modernité ou une conséquence de l'évolution des techniques. De la sphère privée, l'intimité devient collective. Si la pudeur et la dignité restent l'apanage du singulier, chaque homme est obligé de revendiquer collectivement pour être reconnu dans sa dignité originelle qui fonde I'humain. Que cela soit accepté et reconnu au plus haut niveau serait déjà un premier pas vers une société où chacun pourrait à nouveau se reconnaître, un premier pas vers le bien-être de la population dans son ensemble. $(2009$, p. 3)

Il y aurait donc une forme de reconnaissance entre l'écrivain et le lectorat. Alan Pauls explique cette relation complexe : « une voix [celle de l'écrivain] qui nous frappe soudain par son ignoble nouveauté » contre "ces yeux posés sur nous [ceux du lecteur], étonnés, nous glacent les sangs » (p. 38). Chaque mot mis sur le papier devient alors un risque. Chaque mot, selon sa place, sa forme, son enchaînement et sa signification, au sein d'un texte formant un tout (à l'image de la plage formée de grains de sable), donnera une impression particulière, révélatrice de l'intimité de l'écrivain et créatrice d'un jugement chez le lecteur. Ce sont les mots qui déterminent la force de chaque œuvre, c'est pourquoi l'analyse de l'écriture du souvenir est primordiale pour mener à bien la réflexion.

Écrire l'intime, c'est aussi écrire à propos des expériences personnelles, des aventures de la vie, des lieux qu'on a visités, des images qu'on a vues, des sons qu'on a entendus. Autrement dit, l'écriture du souvenir commence par un éveil des sens. 
Alan Pauls fait appel à l'éveil des sens multipliés, qui évoque soit un sentiment positif: « l'omnisprésence des pins dans les forêts, de la viande de cerf fumée ou du goulasch au menu des restaurants, de l'architecture alpine [...] et les salons de thé, le bois sombre et les toits pentus, les rideaux volants » (p. 25), soit un sentiment négatif: « la mer, devenue noire [...], agitée, sale et transportant des tonnes d'écume iodée qu'elle déposait sur le bord, pour qu'elles se souillent [...] d'algues, de poissons morts, de restes de mollusques et de nourriture, de cadavres de mouettes et de phoques » ( $p$. 93). Il insiste aussi sur le caractère érogène de la plage qui réside dans la vue plus que dans le toucher : « la plage n'est jamais érogène quand elle se mêle au corps [...] mais l'est au contraire lorsque le contact physique avec la chair [...] est remplacé par un contact visuel » (p. 56). C'est d'ailleurs pourquoi il se concentre sur les textures, les couleurs et les formes pour décrire le paysage qui l'entoure selon les images et sensations scellées dans sa mémoire. Il a créé dans son esprit un album photo, support qu'il utilise comme modèle dans son ensemble et qui survient dès le début, dans la scène du cinéma drive-in : «je me rappelle tout : la carte postale imaginaire de notre petite voiture aux phares éteints » (p. 17). Les souvenirs sont conservés grâce à une capacité à enregistrer des images mais ne peuvent pas être retranscrites de la même manière en écriture, d'où le décalage entre l'affirmation de Pauls « je me rappelle tout » et les informations limitées données au lecteur. Mitchell dans «There are no visual media ", souligne le fait qu'il n'y ait pas de médias purs et cela peut se déporter sur le processus de l'écriture directement (Mitchell, 2005), comme le prouve la composition de la plage donnée par Pauls : «Les plages les plus pures ne le sont jamais davantage que le sable qui les forme, et le sable est tout sauf pur » (p. 31). La seule pureté, ou pourrait-on dire la seule authenticité, que semble donner le récit intime serait donc la notion de temporalité puisqu'il affirme le passé. Cependant, pour Pauls, cela ne s'avère pas si simple. II fait remonter son autobiographique à un passé qu'il n'a pas connu et cela toujours en relation à la plage : « Antium était le Palm Beach de Caligula, Néron et autres joyeux bipolaires de l'Antiquité » (p. 45) et en relation à la littérature: «Sénèque et Pline le Jeune ébauchaient déjà, d'une certaine façon, l'esthétique existentielle que Foucault revendiquerait au début des années quatre-vingt » (p. 45). Il créé donc un lien alambiqué entre passé et présent, où le souvenir joue un rôle unique qui consiste à « ajouter de [la] splendeur au passé et qu'aucun présent ne confirmera jamais » (p. 17). Mais l'écriture du souvenir ne s'arrête pas là. Elle est également assortie du concept d'étrangeté, développé par Freud, et repris par Pauls à travers la séparation entre la plage impliquant l'altérité, la nouveauté, la sauvagerie et le monde de la ville « où tout est trop familier, trop 
docile » (p. 99). Similairement, l'écriture du souvenir relève de ce qui est étranger, de ce qui est autre, lorsqu'il s'agit de mettre de côté sa pudeur. Camenen mentionne l'excentricité dont Alan Pauls témoigne pour raconter le passé décrit comme des " gisements de la mémoire » et précise que cette étrangeté " devient alors une expérience, intime, de lecture » (Camenen, 2012). Il y a un retour sur la relation entre l'écrivain et son lectorat, dont Pauls a conscience car il est lui aussi lecteur. C'est pourquoi il mentionne d'autres textes autobiographiques, dont il a fait l'expérience en tant que lecteur, entre autres le Journal de Witold Gombrowicz, dont une citation est insérée à la page 53. Cette démarche revient à questionner la valeur de l'autobiographie en comparant la nudité physique à l'idée de se mettre à nu (écrire son histoire). Il mentionne aussi les mémoires de John Updike, qu'il cite également : «Et le poids du soleil sur ma peau signifiait toujours ceci pour moi: j'étais en train d'être rédimé, de rejoindre le genre humain, laissant derrière moi difformité et honte», écrit-il dans ses mémoires » (p. 109). Deux aperçus sont ici donnés : premièrement, la filiation entre l'écriture du souvenir et l'appartenance au genre humain. Dire le passé, c'est affirmer son existence impactée dans le présent de l'écriture et le présent du monde en général (dire d'où l'on vient pour savoir où l'on va). Secondement, la plage devient plus que l'objet d'un souvenir, elle devient une expérience, ce que Pauls répartit sur quarante ans et fragmente en plusieurs catégories : « la joyeuse carbonisation », «l'indifférence adolescente », « la prudence», «le scrupuleux management solaire », « la protestation » et « la résignation » (p. 108). II donne pour exemple la cigarette allumée sur la plage, où pour protéger la flamme du vent, il faut l'entourer de ses mains. Ce geste devient une habitude, un reste de l'expérience de la plage (« Nous appelions cela une «expérience»», p. 98), qu'il exécute même quand il se trouve à l'intérieur. II parle de «lapsus de plage » (p. 98). L'écriture du souvenir n'est donc pas délimitée, tout simplement parce qu'elle doit travailler avec la matière psychique humaine. Elle se place dans une zone ouverte et illimitée, qu'il est impossible de réduire aux mots uniquement. En réponse à cette pluralité, Alan Pauls sollicite fortement le monde visuel, pour illustrer (littéralement et métaphoriquement) son texte-essai et ses souvenirs.

\section{Le culte de l'image}

Le dernier point de réflexion gravite autour du culte de l'image. Alan Pauls choisit d'insérer dans son texte des images, au sens propre et figuré, parce que «Texte et image, loin de s'illustrer, se transforment l'un l'autre, jusqu'à donner forme à des compositions 
d'un type nouveau, faites d'unités qui, d'une certaine façon, ont perdu leur indépendance » (Baetens, 2013, p. 261). Sa démarche reprend bien l'idée de pluralité. Le texte polymorphe devient le lieu idéal d'expression de l'intime, qui a tout autant voire plus de formes, comme cela a déjà été observé. La première trajectoire qu'adopte le récit est sûrement la plus évidente puisqu'il s'agit du visuel en action dans le texte. Tout au long du livre apparaissent de nombreux détails, au sein du texte lui-même, qui se concentrent sur l'aspect graphique et titillent aussi bien l'esprit que l'œil du lecteur. C'est le cas par exemple de l'usage des parenthèses, pour transmettre son sens de l'humour et exprimer la notion de littéralité : « je choisis le sable (et je mets l'eau entre parenthèses) » (p. 111), ou pour créer un effet particulier : « (Je crains qu'il n'y ait que deux façons de se distinguer sur la plage: [...] » (p. 38), parenthèse qui ne se ferme qu'une page plus loin, avec un tout autre sujet : « montra du doigt en souriant la ballon qui fuyait au loin). » (p. 39). Ce procédé traduit deux effets. Le premier est en rapport avec son caractère : Pauls apparaît comme celui qui se perd dans de longues digressions, volontaires ou non. Le second est en rapport avec la valeur du texte : en mettant entre parenthèses un épisode autobiographique, quelle en est sa place dans l'œuvre ? Quel rôle joue-t-il par rapport aux autres épisodes, qui ne semblent pas bien différents et qui pourtant ne sont pas entre parenthèses ? Une réponse probable est qu'il constitue principalement un effet visuel, parce que le paragraphe se termine à la fin de la page, par une parenthèse fermée, et que le lecteur est alors tenté de revenir visuellement en arrière sur le texte pour retrouver l'endroit où elle a été ouverte, à la manière d'un rembobinage. Un des autres aspects visuels réside tout simplement dans la matérialité typographique du texte, notamment à travers l'abondance de mots en italique, l'insertion de pages blanches, l'aspect de bloc de chaque partie ainsi que l'absence de titres ou de désignations (chapitres, numéros) pour les différentes parties du texte. Ces pratiques sont représentatives de la psychologie de l'auteur qui transmet à la fois une impression de prodigalité et de dépouillement. II emploie également un procédé d'ekphrasis pour rendre les images précises de ce qu'il décrit, c'est le cas par exemple lorsqu'il parle d'« une délicieuse douleur, une extase, le type de plaisir sublime que la plage ne fournit que lorsque deux corps brûlés au soleil se glissent dans un lit à peine fait en s'enlacent dans ce paradis propre, frais et simple, de draps de coton blanc » (p. 65). Pour rendre ses descriptions encore plus visuelles, il emploie également la paraphrase, afin d'intensifier les images virtuelles et d'exciter l'imagination du lecteur : " une explosion sexuelle collective, une partouze sauvage généralisée, une bouffée de violence mortelle» (p. 49). Plus encore, il procède à des mises en abyme visuelles. Le meilleur exemple est celui de I'homme sur la plage qui l'imite : « sans dire un mot, [il] fit un geste qui, aujourd'hui 
encore, me laisse sans voix: il m'imita l'imitant » (p. 79). Cette mise en abyme, mise en valeur par l'italique, montre à quel point l'image de l'autre dans son altérité peut avoir un impact sur la psychologie. L'homme ne prononce pas une seule parole et pourtant, Pauls se souvient encore de la scène après plusieurs années et la seule façon qu'il a d'en parler, c'est de l'écrire. Les images semblent avoir une conséquence directe sur la psychologie humaine, puisque Pauls précise que « Nous y allions pour être uniques, pour pouvoir, six mois ou un an plus tard, exhumer les photos de ce moment difficile et réaffirmer combien nous étions exceptionnels, en nous délectant du spectacle de nos silhouettes, uniques, découpées sur fond de plage déserte » (p. 94). Ainsi, tout comme la plage, la photographie renvoie une image altérée du moi. Sur une photographie, chacun peut être qui il veut, il n'y a pas d'identité mais juste un moment figé, capturé sur le papier. On comprend donc bien pourquoi Alan Pauls a choisi d'insérer des photographies d'enfance à la fin de chaque partie. Non pas pour illustrer ce qu'il vient de raconter, mais pour nous faire voir un enfant, une plage, un adolescent, couché, debout, assis ou lisant un livre, faisant un château de sable, etc., afin de pouvoir créer un réseau d'images mentales, venant se poser additionnellement à côté du texte. C'est d'ailleurs pour cette raison que Pauls a sélectionné des photos qui n'ont rien à voir avec les épisodes qu'il reporte, elles sont dénuées de précision, mais elles ne sont pas dénuées d'histoire, ce qui laisse penser que Pauls n'a pas tout dit (et ne peut pas tout dire). Dans un article sur la photographie dans le récit autobiographique, Véronique Montémont explique que « texte et photo partagent un matériau commun, l'histoire individuelle, qu'ils finissent par exprimer, assez logiquement, dans un même lieu » $(2008, \$ 5)$. Ce même lieu, c'est le livre. Mais pourtant, le moyen de transmission ne semble pas être exactement le même. En effet, afin de fonctionner harmonieusement dans le genre autobiographique, la photographie doit «s'autodésigner par un acte de langage » (Montémont, 2008, §18). Ainsi, la photographie est complétée par le texte et le texte est complété par la photographie. Il ne s'agit plus de simple rencontre entre les deux mais bien d'un discours multi-énonciatif, qui donne naissance à des intrusions narratives implicites. Il y a immixtion. De plus, la photographie prend pour destinataire l'auteur lui-même et pas seulement le lecteur-spectateur. Jacques Roubaud souligne le fait que

La photographie de nous enfant nous fascine ; parce qu'elle nous montre une scène où nous étions présents; nous voyons que nous $y$ étions; nous nous y reconnaissons; or nous ne nous souvenons pas de cette scène ; nous n'en n'avons rien vu. [...] La photographie me montre la première forme de l'invisible : celle de l'oubli. (Roubaud, 1993, p. 251) 
L'écriture intime prend donc un autre tournant dès qu'elle est associée à la photographie. Une recomposition d'un passé perdu est alors possible. L'univers d'adolescent d'Alan Pauls est retrouvé dans une médiation de lui à lui-même.

Ces aspects visuels en action dans le texte sont prolongés dans un deuxième temps par l'importance fondamentale du cinéma. Dès la première page, le monde du cinéma se mêle au monde des rêves à travers le psychisme d'Alan Pauls, qui le partage à la manière d'un pacte avec son lecteur : «Mettons que chaque rêve est un film » ( $p$. 9) et inversement, le monde du rêve s'immisce dans le monde du cinéma : « les rêves à l'affiche durant l'été 2005 furent particulièrement nombreux » (p. 9). Dès le départ, il place le lecteur dans un espace psychologique multiforme. Cette proximité rêvecinéma suit sa progression jusqu'au point où le cinéma ne relève plus uniquement de l'intériorité de l'auteur, mais directement de ses expériences de la vie. Il y a beaucoup de références directes à de nombreux films, mais les plus saisissantes concernent des moments clés de la réalité de l'existence, tout particulièrement en rapport à la sexualité et la violence, et qui sont donc susceptibles d'influencer sa psychologie. Premièrement, la plage est signalée comme lieu de rejet de la sexualité à travers l'expérience cinématographique : « La plage n'est jamais aussi érotique que lorsque James Bond, caché derrière un palmier, voit la déesse marine Honey Rider [...]. Mais l'intensité de cette épiphanie est gâchée quelques minutes plus tard [...] quand, [...] les mêmes personnages cèdent au désir » (pp. 56-57). Secondement, le cinéma accompagne l'expérience de la guerre. Pauls reporte à nouveau la rencontre entre James Bond et Honey sur la plage, scène qui parodie une situation de débarquement colonial menant à l'affrontement du colonisateur et de l'indigène séduisante. Mais quelques lignes plus tard, il précise que «Bien des années plus tard, [il assistait] sur une plage proche de La Havane à une version plus crispée de la même scène : sur la côte, des canons enterrés dans le sable » (p. 59). Son mode de compréhension et de perception de la violence de la guerre est prévenue par l'expérience cinématographique initiale. On retrouve également le phénomène inverse : « $\mathrm{Au}$ fond, tout ce que je sais des drive-in, je l'ai appris par la suite, au cinéma » (p. 15). II traverse en premier lieu une expérience existentielle, ici celle de la connaissance, puis l'expérience cinématographique intervient par la suite, toujours comme élément entremetteur. Selon ces considérations, l'écriture et le cinéma partagent la même caractéristique : ils jouent tous deux un rôle médiateur entre la réalité et la psychologie. De ce fait, l'écriture intime de Pauls se teinte doucement et devient écriture cinématographique, tout particulièrement dans la manière qu'il a de poser le 
décor d'une scène : " un plan serré : moi, assis dans la voiture, sur le bord du siège arrière [...] mon père se tournait, de profil sur le siège avant » (p. 18). De surcroît, la page 55 met en place un horizon nouveau, où l'adolescent n'est plus personnage, mais acteur, vivant littéralement l'expérience du tournage : "le sergent Warden et Karen Holmes recommencent à s'embrasser, allongés sur une plage de Hawaï [...] je ne puis m'empêcher de penser à ce sable désagréablement mouillé, dur », où la scène devient mise en scène : "Fred Zinnemann décida d'interrompre la prise », et où le romantisme devient fragmentation : "une légion d'insupportables petits cristaux ». Au même moment, Alan Pauls devient réalisateur et son livre devient film. Mais il n'en reste pas moins une œuvre littéraire et une telle juxtaposition des deux arts engage une réflexion qu'encore une fois, Pauls décide de réaliser à travers la plage. Tout d'abord, elle lui permet de justifier l'écriture de La vie pieds nus, lorsqu'il explique qu'il $y$ a des centaines de films sur la plage, mais que seulement très peu de livres s'y consacrent (« il existe peu de livres sur la plage, mais combien de livres de plage les libraires recommandent-ils » (p. 87)). Ensuite, elle lui permet de justifier son statut d'écrivain, dont le passage suivant, sur le film Julia, en est l'illustration :

Oui, je crois que c'est avec Julia - c'est-à-dire en 1976 : j'étais alors un jeune apprenti écrivain prêt à suivre immédiatement n'importe quel protocole vaguement convaincant qui me permît de me forger une personnalité littéraire - que j'appris à idolâtrer, de façon assez inexplicable je dois dire, ce paradis étrange, inconfortable, âpre et hostile que devenait la plage lorsqu'elle tombait aux mains de l'imaginaire littéraire ou intellectuel. (p. 90)

Le film semble l'avoir guidé d'abord dans son expérience de l'écriture, puis plus généralement dans sa carrière d'écrivain. C'est pourquoi Pauls mentionne tout au long du livre de nombreux réalisateurs et œuvres cinématographiques qui semblent avoir influencé son écriture de l'intime, de la même manière que cet exemple-ci. Mais ce culte de l'image, aussi grandissant soit-il, se renverse au profit d'une nudité absolue, elle aussi source d'inspiration.

Après toutes ces images, cette abondance visuelle, ce festival des formes et contenus, Alan Pauls embrasse, dans toute sa splendeur, le pouvoir du vide. Cet empire du néant se décline selon plusieurs niveaux qui, loin d'épuiser l'écriture intime, lui apporte une richesse singulière. Avant tout, c'est bien entendu la plage qui 
est dépeinte comme la résidence première du vide : « la plage est un territoire vide de toute image [...] [y réside entièrement cette] sorte de chasteté iconographique [...] Le sable et la mer supportent mal le caractère actuel des images, non leur puissance » (pp. 11-12). La plage est donc le meilleur endroit pour rêver, se perdre dans ses pensées voire même pour l'expérience hallucinatoire. Elle aussi lieu de nudité. D'un côté de la nudité des corps: « le seul espace public où la nudité presque complète n'est ni une exception ni une provocante infraction, mais un principe de coexistence, un style de vie, une loi » (p. 43), et de l'autre, de la nudité comme essence de la plage elle-même : « la plage est un espace nu, [...] ce dépouillement complet [...] a un corollaire moral presque immédiat » (pp. 33-34), et par conséquent, lieu où l'écrivain peut réfléchir à sa propre vie et peut-être même sa mort, dans sa relation aux autres et dans sa relation à soi, éveillant ainsi une réflexion d'ordre moral (approbation ou remise en cause de son comportements, ses actions, ses traits de caractère, etc.). Il se met à nu, physiquement et psychologiquement. II y a un phénomène d'évaporation de la conscience présente, pour se plonger dans une conscience plus profonde, déconnectée du hic et nunc. Parce que la plage est vide et blanche, elle permet un décalquage de notre propre vie. Elle agit comme une toile vierge sur laquelle on peut contempler son existence. En relation avec le cinéma, Pauls opte pour l'idée de l'écran blanc: « le spectacle, le vrai, le seul que l'univers de la plage ne rejette pas comme une chose redondante ou vexatoire, était celui de l'écran blanc, sorte de cinéma vierge, passif, qui ne fascinait pas en raison de ce qu'il dégageait mais de toutes les images qu'il parvenait à suggérer » (p. 18). En d'autres termes, l'écran blanc débride l'esprit. II permet une liberté complète de l'imagination, qui semble bien plus fructueuse que les images évoquées par d'autres images déjà existantes. L'écran blanc est un affranchissement psychologique, une délivrance artistique, donnant littéralement « carte blanche » à l'écrivain. II brise le déterminisme. C'est en ce sens que la plage passe de la matérialité (écran blanc) à une abstraction qui résiste au temps:

la plage - toute plage - est toujours vierge [...] peu importe qu'elle soit loin de son passé primitif et colonisée par la modèle capitaliste d'exploitation du temps libre. Si elle est toujours vierge, c'est parce que cette virginité n'est plus un état naturel susceptible d'être préservé ou dégradé, entretenu ou altéré, mais un concept. Aujourd'hui plus que jamais, la plage trouve dans la virginité un facteur autrement plus significatif qu'un simple état de perfection : elle trouve son Idée. (p. 22) 
La plage est donc un instrument pour interpréter le temps, le passé de l'enfance, et un instrument pour le conceptualiser. La notion de vide intervient également dans l'interprétation des relations humaines. Le figure du père par exemple : « mon père, Allemand arrivé en Argentine à six ans et jamais naturalisé argentin, bien qu'à quatorze il connût les rues de Buenos Aires, le dialecte du Río de la Plata et la composition des différentes équipes de football locales [...] sans doute par une sorte de réflexe d'adaptation excessif » (p. 23), qui se transforme en toile blanche, sur laquelle ont été dessinés, collés, assemblés, des éléments identitaires éclectiques. Mais c'est aussi le cas de l'adolescent lui-même, qui devient écran blanc, par héritage paternel mais aussi par sa tendance à se distinguer des autres : " la honte que m'inspirait ma peau, si blanche, si sensible et si faible [...] sur cette page peuplée de Noirs » (p. 105). Dernièrement, le vide réside dans I'histoire elle-même et la dernière partie du livre en est représentative. Cette dernière partie commence par ces mots : «Dans cette scène il y a un enfant ». À la lecture de ce dernier fragment, le lecteur est déboussolé par la présentation d'une scène nouvelle, détachée et qui semble plus réelle que tout ce qui vient d'être dit. Comme si tout ce qui avait précédé était du vide, comme si I'histoire ne faisait que commencer. Alan Pauls se serait donc jouer de nous et nous fait réaliser que nous lisions, d'une certaine manière, notre propre histoire à travers lui : « le livre qu'il vient d'ouvrir et dont le piège se referme déjà sur lui, un piège qui ne se rouvrira plus » (pp. 116-117). II nous fait réaliser que pendant un moment, pendant ce moment de lecture, nous avons nous aussi flouté les traits, perdu les couleurs et nous sommes partis ailleurs, quelque part dans la pensée : « II comprend que ce livre est un autre lieu » (p. 117). Nous avons fait le vide pour revivre nos propres souvenirs d'enfance et réfléchir sur notre propre existence, sans s'en rendre compte. C'est d'ailleurs pourquoi Pauls termine son récit en incluant le lecteur par l'utilisation du pronom personnel « nous » : «les jours de notre enfance que nous vécûmes le plus intensément sont ceux que nous pensions avoir laissé filer sans vivre » (p. 117) et révèle le pouvoir de la littérature, comme lieu de l'intime: " [les jours] que nous passâmes en compagnie du livre pour lequel plus tard, une fois complètement oublié, nous serions prêts à tout sacrifier» (p. 117).

\section{Conclusion}

La vie pieds nus est un bijou philosophique. A mi-chemin entre l'essai et l'autobiographie, cette œuvre permet de repenser au passé, au présent et au futur aussi bien de la vie personnelle que de la littérature. Alan Pauls, comme homme et comme 
écrivain, offre un décor de plage pour ses lecteurs et lui-même, afin de contempler et d'explorer les méandres de la pensée intime et collective. L'écriture intime travaille à l'expression d'une identité, reliée aussi bien à son héritage latino-américain, qu'à son identité littéraire et son identité en tant qu'individu sui generis. En parallèle, elle s'ouvre sur une métalittérature, communiquée par la veine métaphysique, le passage de l'intime au collectif et l'écriture du souvenir. Ces emboîtements complexes sont réalisés à travers un réel pèlerinage, un plaisir presque « chrétien » (comme l'écrit Pauls luimême) de l'expérience visuelle, d'abord en action dans le texte, puis dans l'importance accordée au cinéma et enfin par le pouvoir du vide. En résumé, ce qui se dégage de cette œuvre, n'est rien d'autre que la tentative de dire l'ineffable. On peut conclure sur une citation d'Allain Glykos, qui convient parfaitement à l'univers maritime d'Alan Pauls : « J'écris parce que la réalité, ce que je vis, déclenche en moi des mots, des phrases, à la manière dont les seiches, par exemple, sécrètent de l'encre pour se protéger, se dissimuler. Je sécrète, à ma façon, de l'encre » (Glykos, 2009, p. 28).

Subvention : L'auteure n'a reçu aucun soutien financier pour ce travail.

\section{Bibliographie}

Aubague L., Franco J., \& Lara-Alengrin A. (2009). Les littératures d'Amérique Latine au XXe siècle: une poétique de la transgression? Paris, FR: L'Harmattan.

Baetens J. (2013). Une vie en cartes postales? Poétique 2(174), 261-271.

Bell-Villada G.H. (1999). Borges and His Fiction: A Guide to His Mind and Art. Austin, USA: University of Texas Press. Camenen G. (2012, May 31). Alan Pauls, l'écriture ou l'arène de l'intime. Raison publique. http://www.raisonpublique.fr/article539.html. Consulté le 15 janvier 2019.

Camus A. (1942). L'Étranger. Paris, FR: Gallimard.

Carel A. (2004). L'intime, le privé et le public. Le secret, la discrétion et la transparence. In J. L. Graber (dir.), L'enfant, la parole et le soin (pp. 87-94). Toulouse, FR: Eres.

Glykos A. (2009). Ecriture et transmission. Le journal des psychologues 9(272), 26-29.

Goetgheluck D., \& Conrath P. (2009). L'intime et le collectif. Le journal des psychologues 9(272).

Graber J. L. (2004). Du bon et du mauvais usage du secret. In J. L. Graber (dir.), L'enfant, la parole et le soin (pp. 4765). Toulouse, FR: Eres.

Millet R. (2008). L'arène de l'intime [Interview]. La femelle du requin (30), 60-69.

Mitchell W. J. T. (2005). There Are no Visual Media. Journal of Visual Culture 4(2), 257-266.

Montémont V. (2008). Le pacte autobiographique et la photographie. Le français aujourd'hui 2(161), 43-50. https:// www.cairn.info/revue-le-francais-aujourd-hui-2008-2-page-43.htm\#pa12. Consulté le 17 janvier 2019.

Pauls A., Raynaud V. (trad.) (2007). La vie pieds nus. Paris, FR: Christian Bourgois.

Roubaud J. (1993). La Boucle. Paris, FR: Seuil. 